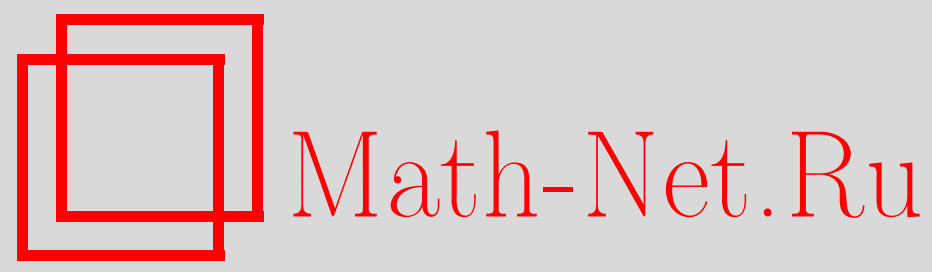

А. П. Старовойтов, Замечание к одной проблеме рациональной аппроксимации, Матем. заметки, 2003, том 74, выпуск 3, 446-448

DOI: https://doi.org/10.4213/mzm278

Использование Общероссийского математического портала Math-Net.Ru подразумевает, что вы прочитали и согласны с пользовательским соглашением http://www.mathnet.ru/rus/agreement

Параметры загрузки:

IP: 54.224 .187 .69

26 апреля 2023 г., 15:25:19

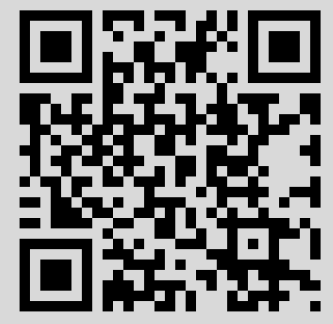




\title{
ЗАМЕЧАНИЕ К ОДНОЙ ПРОБЛЕМЕ РАЦИОНАЛЬНОЙ АППРОКСИМАЦИИ
}

\section{А. П. Старовойтов}

\begin{abstract}
В работе показано, что для любой невозрастающей и сходящейся к нулю числовой последовательности $\left\{a_{n}\right\}_{n=0}^{\infty}$ существует непрерывная $2 \pi$-периодическая функция $g$, для которой последовательность ее наилучших равномерных тригонометрических рациональных приближений $\left\{R_{n}\left(g, C_{2 \pi}\right)\right\}_{n=0}^{\infty}$ и последовательность $\left\{a_{n}\right\}_{n=0}^{\infty}$ имеют одинаковый порядок убывания к нулю.

Библиограффия: 4 названия.
\end{abstract}

Пусть $C_{2 \pi}$ - банахово пространство $2 \pi$-периодических непрерьвных на всей числовой прямой функций с равномерной нормой. Через $\mathscr{P}_{n}^{T}$ и $\mathscr{R}_{n}^{T}$ обозначим, соответственно, множество всех тригонометрических полиномов и тригонометрических рациональных функций степени не вьше $n$ с действительньми коэффициентами. Для $f \in C_{2 \pi}$ и $n=0,1,2, \ldots$ рассмотрим $E_{n}\left(f, C_{2 \pi}\right), R_{n}\left(f, C_{2 \pi}\right)$ - наилучшие приближения $f$ в $C_{2 \pi}$ множествами $\mathscr{P}_{n}^{T}, \mathscr{R}_{n}^{T}$.

Ниже соотношение $\alpha_{n} \asymp \beta_{n}$ означает, что бесконечно малые последовательности $\left\{\alpha_{n}\right\}_{n=0}^{\infty},\left\{\beta_{n}\right\}_{n=0}^{\infty}$ имеют одинаковый порядок при $n \rightarrow \infty$, т.е. существуют такие положительные постоянные $A$ и $B$, для которых $A \alpha_{n} \leqslant \beta_{n} \leqslant B \alpha_{n}$ при $n=0,1,2, \ldots$ Если $\alpha_{n} / \beta_{n} \rightarrow 1$ при $n \rightarrow \infty$, то будем писать $\alpha_{n} \sim \beta_{n}$, а запись $a_{n} \downarrow 0$ означает, что рассматривается числовая последовательность $\left\{a_{n}\right\}_{n=0}^{\infty}$, которая не возрастает и сходится к нулю. Например, хорошо известно, что для любой функции $f \in C_{2 \pi}$ последовательности ее наилучших приближений ведут себя именно таким образом, т.е. $E_{n}\left(f, C_{2 \pi}\right) \downarrow 0$, $R_{n}\left(f, C_{2 \pi}\right) \downarrow 0$.

Известна (см. [1]) следующая задача (ослабленный вариант обратной задачи Бернштейна [2] для рациональных приближений).

ПРОБЛЕМА ДоЛЖЕНКО. Какой должсна быть числовая последовательность $a_{n} \downarrow$ 0 , чтобы существовала такая функиия $f \in C_{2 \pi}$, для которой

$$
R_{n}\left(f, C_{2 \pi}\right) \asymp a_{n} ?
$$

При этом (используя терминологию Бернштейна) будем говорить об әффективном решении проблемы в том случае, когда по заданной последовательности $\left\{a_{n}\right\}_{n=0}^{\infty}$ искомая функция построена конструктивно.

В работе [3] доказана следующая 
ТЕОРема 1. Для любой строго убьвающей к нулю последовательности $\left\{a_{n}\right\}_{n=0}^{\infty}$ неотрицательных чисел существует такая нечетная функиия $f \in C_{2 \pi}$, что

$$
R_{n}\left(f, C_{2 \pi}\right)=a_{n}, \quad n=0,1,2, \ldots
$$

Следовательно, если числовая последовательность $a_{n} \downarrow 0$ строго убывает, то она является последовательностью наилучших рациональных приближений некоторой функции $f \in C_{2 \pi}$. Поэтому для такой функции $R_{n}\left(f, C_{2 \pi}\right) \asymp a_{n}$.

На самом деле из теоремы 1 можно вывести следующее

УТВЕРЖДЕНИЕ. Для произвольной числовой последовательности $a_{n} \downarrow 0$ существует такая нечетная функиия $g \in C_{2 \pi}$, для которой

$$
R_{n}\left(g, C_{2 \pi}\right) \sim a_{n}
$$

Действительно, если последовательность $a_{n} \downarrow 0$, начиная с некоторого номера, состоит из нулей, то в качестве искомой функции $g$ можно взять подходящую рациональную дробь. Поэтому предполагаем, что члены последовательности $a_{n} \downarrow 0$ не равны нулю. В этом случае последовательность

$$
\left\{b_{n}\right\}_{n=0}^{\infty}=\left\{\frac{n+2}{n+1} a_{n}\right\}_{n=0}^{\infty}
$$

строго убьвает. Значит, существует такая нечетная функция $g \in C_{2 \pi}$, для которой при $n=0,1,2, \ldots$ вьполнено $R_{n}\left(g, C_{2 \pi}\right)=(n+2) a_{n} /(n+1)$. Следовательно, $R_{n}\left(g, C_{2 \pi}\right) \sim$ $a_{n}$.

Далее рассмотрим следующие последовательности положительных чисел:

$$
\begin{gathered}
\Delta b_{n}=b_{n-1}-b_{n}, \\
\varepsilon_{n}=\min \left\{\Delta b_{1}, \Delta b_{2}, \ldots, \Delta b_{n}\right\}, \quad n=1,2,3, \ldots, \\
\beta_{j}=\frac{\varepsilon_{1}}{5} \frac{\varepsilon_{2}}{5} \ldots \frac{\varepsilon_{j}}{5}, \quad j=1,2,3, \ldots
\end{gathered}
$$

Считая, что $c_{k}=\left(1-\beta_{k}^{2}\right) /\left(1+\beta_{k}^{2}\right), k=1,2, \ldots, n$, определим синус-дроби Чебышева-Маркова:

$$
\nu_{n}(x)=\sin \varphi_{2 n}(x)=\sin \Phi_{2 n}(y)=\sqrt{1-x^{2}} \frac{P_{n-1}(x)}{\prod_{j=1}^{n}\left(1-c_{j} x\right)},
$$

где $P_{n-1}(x)$ - алгебраический полином степени $n-1$ с действительными коэффициентами. Если

$$
t=\left(t_{0}, t_{1}, t_{2}, \ldots\right) \in K=\left\{t: 0 \leqslant t_{k} \leqslant \varepsilon_{k+1}, k=0,1,2, \ldots\right\},
$$

то полагая $\Delta t_{k}=t_{k-1}-t_{k}$, рассмотрим функцию

$$
f_{t}(\theta)=\sum_{k=1}^{\infty}\left(\Delta b_{k}+\Delta t_{k}\right) \sin \theta \frac{P_{k-1}(\cos \theta)}{\prod_{j=1}^{k}\left(1-c_{j} \cos \theta\right)} .
$$


В работе [3] доказано, что при любом $t \in K$ функция $f_{t} \in C_{2 \pi}$ и подчинена условиям

$$
b_{n}+t_{n}-\varepsilon_{n+1} \leqslant R_{n}\left(f_{t}\right) \leqslant b_{n}+t_{n}, \quad n=0,1,2, \ldots
$$

Поэтому полагая $g=f_{t^{*}}$, где $t^{*}=\left(\varepsilon_{1}, \varepsilon_{2}, \varepsilon_{3}, \ldots\right) \in K$, и учитывая свойства последовательностей $\left\{\Delta b_{n}\right\},\left\{\varepsilon_{n}\right\}$, получим, что при $n=0,1,2, \ldots$

$$
\frac{n+2}{n+1} a_{n} \leqslant R_{n}\left(g, C_{2 \pi}\right) \leqslant 2 \frac{n+2}{n+1} a_{n} .
$$

Следовательно, $R_{n}\left(g, C_{2 \pi}\right) \asymp a_{n}$.

Таким образом, мы показали, что справедлива следующая теорема, дающая окончательное (эффективное!) решение проблемы Долженко.

ТЕОрема 2. Для произвольной числовой последовательности $a_{n} \downarrow$ с существует такая нечетная функиия $g \in C_{2 \pi}$, для которой

$$
R_{n}\left(g, C_{2 \pi}\right) \sim a_{n}
$$

Кроме того, для произвольной числовой последовательности $a_{n} \downarrow 0$, все члень которой отличны от нуля, нечетная функиия (1) при $t=t^{*}$ удовлетворяет условию

$$
R_{n}\left(f_{t^{*}}, C_{2 \pi}\right) \asymp a_{n} .
$$

Как известно, в полиномиальном случае проблема эффективного построения функции с заданными наилучшими полиномиальньми приближениями поставлена еще Бернштейном (см. [4, с. 294]) и до сих пор не решена. Более того, в полиномиальном случае для заданной последовательности $a_{n} \downarrow 0$ в настоящее время отсутствуют эффективные примеры непрерьвных функций, для которых

$$
E_{n}\left(g, C_{2 \pi}\right) \asymp a_{n}
$$

\section{СПИСОК ЦИТИРОВАННОЙ ЛИТЕРАТУРЫ}

[1] Долженко Е. П. Сравнение скоростей рациональной и полиномиальной аппроксимаций // Матем. заметки. 1967. Т. 1. № 3. С. 313-320.

[2] Бернштейн C. H. Sur le problème inverse de la théorie de la meilleure approximation des fonctions continues // C. R. Acad. Sci. 1938. V. 206. P. 1520-1523.

[3] Старовойтов А. П. К проблеме описания последовательностей наилучших тригонометрических рациональных приближений // Матем. заметки. 2001. Т. 69. № 6. С. 919—924.

[4] Бернштейн С. Н. Собрание сочинений в 4-х томах. Т. 2. М.: Изд-во АН СССР, 1954. 